\title{
The Major Ruling Role of our Weak Nonlinear Electromagnetic Field
}

\author{
Maria Kuman* \\ Holistic Research Institute, USA
}

Submission: August 25, 2018; Published: October 10, 2018

*Corresponding author: Maria Kuman, Holistic Research Institute, 1414 Barcelona Dr., Knoxville, TN 37923, USA;

Email: holisticare@mariakuman.com

\section{Abstract}

The article accentuate on the important major role, which the weak nonlinear electromagnetic field (NEMF) plays in the human body - it rules and regulates all biochemical processes in the body. This is because all biochemical substances have weak electromagnetic fields and are influenced by external weak electromagnetic fields (EMF). Since all organs are subordinated to the Subconscious, the human NEMF rules and regulates all the processes from the Subconscious. It operates with the waves of the NEMF as a quantum computer. Measurements of the author, when combined with Russian measurements reveal the dynamic of the human NEMF, which is ruled by emotions. These combined studies reveal the essential role emotions play in our health and wellbeing.

Keywords: Human nonlinear electromagnetic field; How NEMF rules and regulates; NEMF as a quantum computer; Dynamic of NEMF; Emotions rule NEMF

\section{Introduction}

I spent almost 40 years of my life measuring the human weak NEMF. It is 1,000 times weaker than the biocurrents of the physical body. While the biocurrents are measured in milliamperes, the weak nonlinear electromagnetic field (NEMF) is measured in microamperes and parts of them. Since measuring NEMF required very sensitive equipment, NEMF remained terra incognita for many many years. Being unable to measure it, most of the scientists denied it exists.

It was found that very weak currents (microamperes) can stimulate bone regrowth [1], but there was no understanding why. It was also found that all cancer cells are electrically negative but there was no understanding why. It was found that the intracellular space is mostly water and collagen and they both are very sensitive to electrical changes and so are the membranes between the cells.

It was also found that cellular populations are effective accumulators of photons and very sensitive to photon emission with wavelength from 350 to $800 \mathrm{~nm}$. It was found that the DNA, RNA, and all other bioactive substances have EMF, emit weak NEMF (including photons) [2,3], and are sensitive to weak NEMF (and photons). Based on study of the weak photon emission, F. Popp [3] concluded in 1983 that the super-weak field in the organism is capable of regulating all biochemical processes (through electromagnetic interaction).

\section{Where is the NEMF Located?}

Our brain has two hemispheres. While the left hemisphere is specialized in: writing, reading, reasoning, mathematics, speech and languages, etc., which relate to the conscious, the right hemisphere is specialized in: spacial imaging, visualizing, artistic ability, etc. which relate to the Subconscious [4,5]. Many scientists think that the right hemisphere relates to the subconscious and the left hemisphere to the conscious - simple, but not quite true.

Hypnosis, which put the Conscious to sleep and allows access to the Subconscious, revealed that at subconscious level we have a full-life memory storage and that calculations are done with very high speed. This means that at subconscious level we have a sophisticated Quantum Computer, which as shown in a previous article [6], operates with the waves of our NEMF located in the Subconscious.

This Quantum Computer of the Subconscious, through the waves of our NEMF rules and regulates everything in the body. In the Subconscious is also the whole emotional brain consisting of a larger ring related to positive emotions and a smaller ring related to negative emotions. Let us see how positive and negative emotions relate to the surface of the brain hemispheres. If the whole subconscious was projected onto the right hemisphere, all the emotions would be projected onto the right hemisphere, but this is not the case.

Clinical observation on the behavior of epileptic patients show that:

a) when EEG measures enormously high electric potential on the surface of the right-hemisphere of the brain, the patient usually cries for no obvious reason, which means that negative emotions relate to the right hemisphere; 
b) when EEG shows abnormally high electric potential on the surface of the left-hemisphere of the brain, the patient usually laugh for no obvious reason, which means that positive emotions relate to the left hemisphere [3].

Also, a careful study of facial photographs shows that sadness and depression are more intensely expressed on the left side of the face (where the electrical activity of the facial muscles was found to be higher), which is ruled by the right hemisphere. This means that sadness and depression are emotions of the right hemisphere. On the contrary, positive feelings are more intensely expressed on the right side of the face (where the electrical activity of the facial muscles was found to be higher), which is ruled by the left hemisphere [5]. This means that positive emotions are specific for the left hemisphere.

Thus, the larger ring of positive emotions of the Subconscious is obviously projected onto the surface of the left hemisphere, while the smaller ring of negative emotions of the subconscious is projected onto the surface of the right hemisphere. Therefore, the whole Subconscious is not projected onto the surface of one brain hemisphere. Also, clinical finding show that a small stroke in the subconscious could disable a huge portion of cells on the brain surface, which means that the projection is like a funnel.

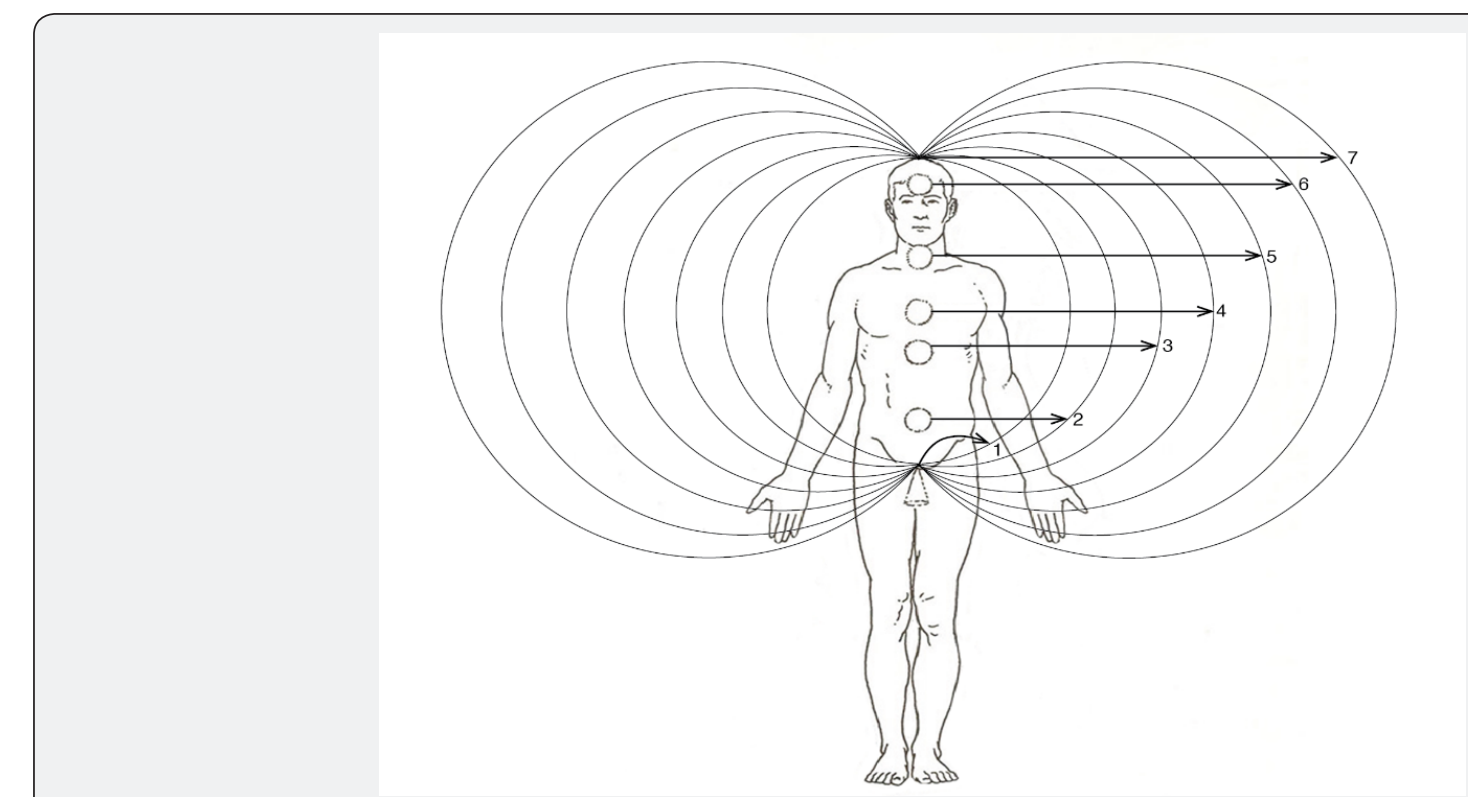

Figure 1: The alternating vortices and anti-vortices of our torus-shaped NEMF.

If you look at the human NEMF on Figure 1, you will see the funnel on top of the head, but it goes deeper. However, the funnel is twisted because the left side of the face and the left arm and leg project onto the right hemisphere and the right side of the face and the right arm and leg onto the left hemisphere. This twisting indicates that the torus shaped NEMF on Figure 1 spins and it initially span clockwise to suck energy in to build the material body (see the next section for details).

This proves that the material body was created under the orchestration of the torus shaped NEMF on Figure 1, which spins. However, as said some scientists simplify the picture and relate the right hemisphere to the subconscious and the left hemisphere to the conscious. If so, the balance of the two hemispheres is also a balance conscious-subconscious. However, considering the real situation of the positive emotions being projected on the surface of the left hemisphere and the negative emotions projected on the surface of the right hemisphere, the balance of the two brain hemispheres means balance of positive and negative emotions.

\section{Measuring the NEMF and Determining its Dynamics}

The human EMF has a torus shape and is nonlinear, just like the solar EMF has a torus shape and is nonlinear. Also, the human EMF has a chain of alternating vortices and anti-vortices just like the sun has chains of alternating vortices and anti-vortices. However, when the vortices and anti-vortices of the Sun are powerful and visible through telescopes as dark spots called solar sports, the vortices and anti-vortices of the weak NEMF need super-sensitive equipment to be detected.

With our patented super-sensitive energy meter, we measured the chain of alternating vortices and anti-vortices (Figure 1), which are along the backbone. The results of the measurements are presented on (Figure 2).

Using our patented supersensitive energy meter, we measured the weak NEMF (Figure 2) and found that at negative emotions (or just negative thinking) the body looses energy. Not only is the total energy of the NEMF lower, it is more imbalanced because the energy of the genetically weak organ drops down maximally. This means that each distress takes us a step farther to a chronic disease or cancer of the genetically inherited weak organ [7]

Our measurements of NEMF (Figure 2) revealed that at positive emotions (or just positive thinking) the energy of the NEMF increases and becomes more balanced. This means that each positive emotion (or just positive thought) is making you healthier [6]. Perfectly balanced NEMF is perfect health. 

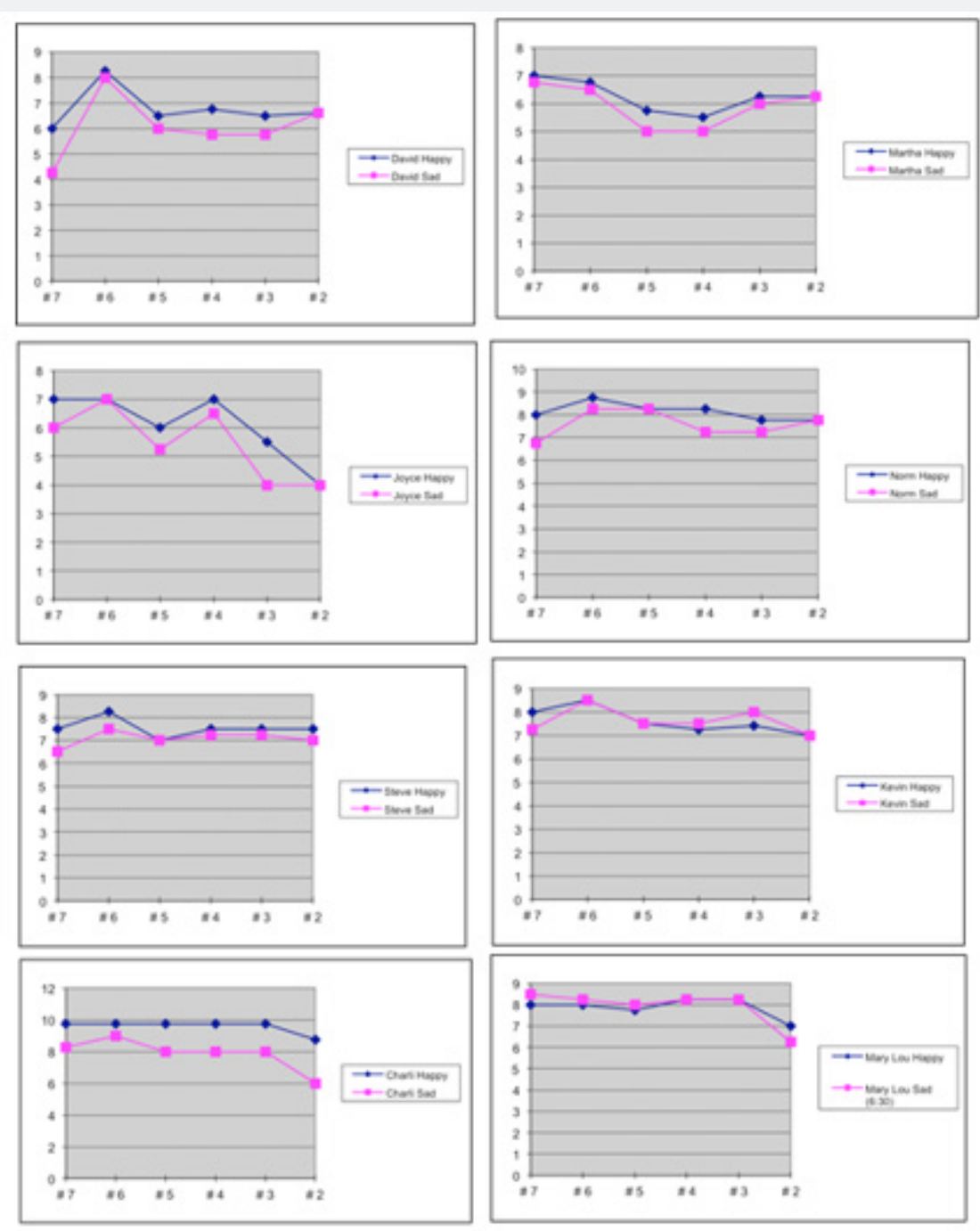

Figure 2: The energy of our NEMF measured at the alternating vortices and anti-vortices pictured on Figure 1.

The Russian scientist Shkatov developed and patented equipment, called 'torsemeter', which allows him to measure the spinning of our NEMF. He found that at negative emotions (distress) the torus-shaped NEMF spins counterclockwise [8]. Also, measurements of Shkatov revealed that at positive emotions the torus-shaped NEMF spins clockwise [8].

Nonlinear Physics needs to step in to explain why things are the way we found them. There is a rule of the folded fingers of the right hand, which states that if the folded fingers were in direction of the electric currents, the thumb would show the direction of the magnetic field induced by the currents. Let us apply this to our spinning NEMF [8].

At negative emotions (or just negative thinking), the counterclockwise spinning of our NEMF will induce counterclockwise currents. Let's orient the fingers of our right hand counterclockwise, and then the thumb would show direction outward for the magnetic field, which means our NEMF will be loosing magnetic energy at negative emotions (or just negative thinking) [8].

At positive emotions (or just positive thinking), the clockwise spinning of our NEMF will induce clockwise currents. Let's orient the fingers of our right hand clockwise, and then the thumb would show direction inward for the magnetic field, which means our NEMF will be gaining magnetic energy at positive emotions (or just positive thinking) [8].

Clockwise should have been the direction of spinning when NEMF enters the fertilized cells because clockwise spinning would bring the energy needed to be sucked in to start the development. Studies in Russia showed that when a generator of torsion fields copied the NEMF of a tadpole and projected it on embryo cell with removed DNA, a tadpole developed [9], which proves that the information carried by the NEMF is the one that rules the development of the embryo.

\section{Conclusion}

If positive emotions make the torus-shaped field spin clockwise and gain energy, while negative emotions make it spin counterclockwise and loose energy, we should be able to compensate for the lost energy at negative emotions with positive emotions. However, ancient Chinese wise men used to say [10]: avoid pursuing big goals in life, but if you fail in pursuing your goal, you need to have 3 to 4 smaller goals the successful achievement of which 
will the positive emotions necessary to compensate for the negative emotions from the failure in pursuing the first goal.

This means that if we have problem balancing our emotions, it is because we need three to four positive emotions to compensate for one negative emotion. Contemporary studies of stress found the same ratio. When comparing the biochemical substances released at negative emotion (distress) with the amount of biochemical substances released at positive emotions, the scientist found the same ratio 1: 3-4 [11].

Now, let's go back to the spinning of our NEMF. If 3 to 4 positive emotions are necessary to compensate for one negative emotion, this means that at negative emotions when the torus-shaped NEMF spins counterclockwise, it must spin 3 to 4 time faster if the lost energy can be compensated only by 3 to 4 positive emotions.

This explains the ancient wisdom of the Hindus "do everything to avoid anger because anger is a very destructive emotion. Always remember that 'Anger' is only one letter away from 'Danger' [7]. A wise man would stop the irritation from growing into anger.

This work is licensed under Creative Commons Attribution 4.0 License DOI: 10.19080/JCMAH.2018.08.555732

\section{References}

1. Becker R (1985) The Body Electric, New York, USA.

2. Garyaev P (1994) Quantum Genome, Moscow, Russia

3. Nagl W, Popp F, Cytobios (1983) 37:45-62

4. Kuman M (1996) A Guide to the Mind's Secrets, Health and Happiness Books.

5. Kuman M (1999) Success in Life through the Power of Mind, Health and Happiness Books.

6. Kuman M (2018) Research in Medical and Engineering Sciences, 5(3).

7. Kuman M (2015) Quantum Mind and Quantum Growth, Health and Happiness Books.

8. Kuman M (2017) Same Fields and Dynamics in Stars and Man, Health and Happiness Books.

9. Tihoplav V, Tichoplav T, Garmonia Haosa (2003) The Harmony of the Chaos, Russia.

10. Veith I, Huang Ti Nei Ching Sue Wen, UK, London,.

11. Actualnie Problemi Stressa, Kishinev, Russia.

\section{Your next submission with Juniper Publishers} will reach you the below assets

- Quality Editorial service

- Swift Peer Review

- Reprints availability

- E-prints Service

- Manuscript Podcast for convenient understanding

- Global attainment for your research

- Manuscript accessibility in different formats

(Pdf, E-pub, Full Text, Audio)

- Unceasing customer service

Track the below URL for one-step submission https://juniperpublishers.com/online-submission.php 